\title{
Percepciones sobre el impacto educativo de la COVID-19: Análisis de un Webinar de la
} Cátedra TECNOEDU.

\section{Perceptions on the educational impact of COVID-19: Analysis of a TECNOEDU Webinar.}

Inmaculada Fernández Esteban. Laboratorio de Educación y Nuevas Tecnologías. Universidad de La Laguna. mesteban@ull.edu.es

Anabel Bethencourt Aguilar. Laboratorio de Educación y Nuevas Tecnologías. Universidad de La Laguna. abethenc@ull.edu.es

Sebastián Martín Gómez. Laboratorio de Educación y Nuevas Tecnologías. Universidad de La Laguna. smarting@ull.edu.es

Cecilia Becerra-Brito. Universidad de La Laguna. cbecerra@ull.edu.es

Manuel Area Moreira. Universidad de La Laguna. manarea@ull.edu.es

\section{RESUMEN.}

El trabajo que presentamos está basado en la experiencia de las actividades de transferencia de conocimiento de la Cátedra Fundación Mapfre de Tecnología y Educación, consistentes en tres webinars donde los ponentes son profesionales de la educación e investigadores en el campo de la Tecnología Educativa. En el público hemos contado con alumnado universitario, de Grado y Posgrado y profesionales de la educación de diferentes niveles educativos. Entre los asistentes se ha difundido un cuestionario para conocer su impresión sobre la situación vivida durante el confinamiento por la pandemia de la COVID-19 y la prospectiva que auguran sobre la educación en relación al uso de la tecnología. Hemos realizado un análisis cuantitativo de variables dicotómicas y cualitativas mediante un sistema de codificación exploratorio, en los dos momentos, durante y después de la pandemia. Además, hemos indagado sobre las posibles relaciones entre códigos, mediante un análisis de coocurrencia. Los principales resultados describen que la mayoría de las personas han dispuesto de los recursos suficientes para afrontar el cambio abrupto a una docencia online, aunque ha supuesto un esfuerzo emocional alto. En cuanto al futuro posCOVID-19, las personas encuestadas consideran que, tras la superación de la pandemia, se abogará por mayor flexibilidad metodológica y didáctica tanto en la oferta de itinerarios en las asignaturas como en la oferta de las titulaciones académicas.

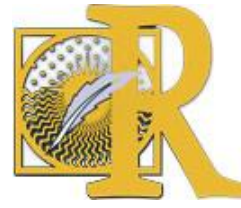


Enseñanza híbrida, tecnología educativa, COVID-19, aprendizaje en línea, webinar.

\begin{abstract}
.
The work we present is based on the experience of the knowledge transfer activities of the Mapfre Foundation Chair for Technology and Education, consisting of three webinars where the speakers are education professionals and researchers in the field of Educational Technology. In the public we have had university, Undergraduate and Postgraduate students and education professionals of different educational levels. Among the attendees, a questionnaire has been disseminated to know their impression of the situation experienced during confinement by the COVID-19 pandemic and the prospects that they predict on education in relation to the use of technology. We have performed a quantitative analysis of dichotomous and qualitative variables using an exploratory coding system, at both times, during and after the pandemic. We have also investigated the possible relationships between codes, through a co-occurrence analysis. The main results describe that most people have had sufficient resources to face the abrupt change to online teaching, although it has involved a high emotional effort. Regarding the future post-COVID-19, the people surveyed consider that, after the pandemic is over, greater methodological and didactic flexibility will be advocated both in the offer of itineraries in the subjects and in the offer of academic degrees.
\end{abstract}

\title{
KEY WORDS.
}

Hybrid instruction, educational technology, COVID-19, online learning, webinar.

\section{Introducción.}

El fenómeno de la pandemia mundial por COVID-19 originó durante el 2020 una situación de emergencia sin precedentes en el ámbito educativo en España. A todos los niveles, este hecho supuso para las instituciones educativas el cese de su actividad presencial y el traslado, tanto de las tareas administrativas como de la práctica docente, a un escenario virtual. Durante el tiempo que duró el confinamiento en los hogares de toda la población, el curso escolar se desarrolló en una modalidad de educación a distancia mediada por tecnología.

La educación a distancia, durante el período de confinamiento, fue condicionada por el uso de dispositivos electrónicos y recursos digitales en el proceso formativo, de manera que para el colectivo docente se tradujo en adoptar y adaptar diversas estrategias metodológicas acordes a cada etapa educativa y en relación al nivel de autonomía y competencia digital del alumnado al que se dirige. En cuanto a la enseñanza universitaria, García-Peñalvo \& Corell (2020) hacen retrospectiva y opinan que ésta pudo llevarse a cabo de manera telemática aparentemente con éxito, con posibles mejoras, pero no sin el empeño (en niveles diversos) de todos los agentes involucrados en el ámbito de la educación. Así pues, ha tenido lugar una experiencia de enseñanza-aprendizaje a distancia que ha destacado vías prometedoras, pero también problemáticas que precisan reflexión.

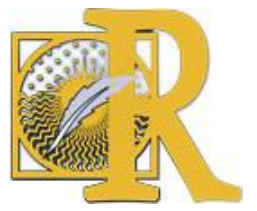


Uno de los efectos negativos de haber adoptado esta modalidad de enseñanza online o a distancia de manera imprevista fue que la brecha digital que se dejaba entrever fuese más evidente aún, ya que se ha manifestado tanto a nivel instrumental como competencial, debido quizás, en parte, al limitado uso que se hacía de estos medios en la enseñanza presencial. El estudio de Kuric Kardelis, Calderón-Gómez, y Sanmartín Ortí (2021) esclarece con mayor profundidad el alcance de esta problemática, haciendo hincapié en tres elementos: el primero es que "la educación online agudiza la desigualdad", el segundo se centra en "la educación virtual como reto del profesorado" y, finalmente, el tercero se basa en "el nivel educativo como factor significativo" (en relación al grado de alfabetización digital). Los trabajos de Cabero y Valencia (2020) y el de Burgos-Videla, Vázquez-Cano, López-Meneses y Adaos Orrego (2020), también abordan esta cuestión a raíz de la experiencia vivida en el confinamiento, haciendo alusión a la brecha digital encontrada entre el profesorado, la cual atiende a la capacidad de saber usar la tecnología con fines educativos en los momentos de nopresencialidad y a las habilidades del alumnado para el uso de las herramientas en su propio proceso de aprendizaje. Estos autores profundizan en el significado de la brecha digital y la fragmentan en tres ámbitos: el acceso a Internet, las necesidades organizativas y didácticas necesarias para una buena experiencia de aprendizaje mediado por tecnologías.

Cabero y Valencia (2020) describen cómo, en el caso del profesorado, la brecha digital está ligada al uso de las herramientas digitales en los momentos de no-presencialidad. Un movimiento muy común es tratar de replicar en un espacio virtual las propuestas didácticas que se usan en la presencialidad, sin una reflexión sobre el proceso de enseñanza y aprendizaje y los nuevos roles. En el caso del docente, ser la figura de transmisión de conocimiento deja de tener relevancia y cobran importancia otras funciones como ser facilitadores del aprendizaje, diseñadores de itinerarios de aprendizaje y tutores virtuales (Cabero y Barroso, 2015).

Un aspecto positivo a destacar que se desprende de esta experiencia de enseñanza mediada por las TIC es que desde el sistema de enseñanza escolar a la universitaria se está recurriendo exponencialmente a metodologías como pueden ser el aula invertida o flipped classroom, ABP (aprendizaje basado en proyectos o problemas), aprendizaje colaborativo online, etc., que han tenido cabida en la educación a distancia y que parecen ser metodologías que facilitan y que tienen su lugar en el aprendizaje mixto, híbrido o semipresencial, también conocido como blended learning, derivado de las condiciones de confinamiento y las medidas restrictivas posteriormente adoptadas a causa de la Covid-19. De hecho, hay múltiples estudios e investigaciones acerca de los beneficios de este tipo de aprendizaje, pero hay una investigación que recoge una explicación del término que data de 2006: "La enseñanza híbrida o blended puede definirse como aquella que combina la instrucción presencial y la instrucción online mediada por las TIC" (Graham, como se citó en Sousa-Santos, Peset-González, y Muñoz-Sepúlveda, 2020,). La misma investigación también ofrece conclusiones acerca de los buenos resultados del sistema flipped classroom y la metodología híbrida en la educación superior. Mucho más reciente, el trabajo de De Obesso y Nuñez (2020), describe la necesidad de una oferta en cuanto a itinerarios y titulaciones que permitan el desarrollo de métodos mixtos. Esto se relaciona también con los

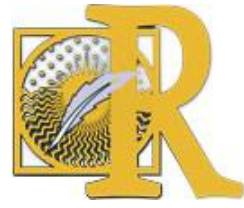


roles cambiantes del docente y del alumnado cuando se desarrolla el aprendizaje en entornos virtuales, que ya presentaban Cabero y Barroso (2015). Estas y otras experiencias y percepciones solicitaban su espacio para ser abordadas en relación al impacto de la Covid19 en la educación.

La presente investigación se desarrolla en el marco de actividad de la Cátedra Fundación MAPFRE Guanarteneme de Tecnología y Educación de la Universidad de La Laguna (TECNOEDU). Esta cátedra tiene como finalidad el desarrollo de actividades de transferencia del conocimiento sobre el uso de las tecnologías digitales en el contexto educativo del sistema escolar y universitario de Canarias. Una de las actividades de la Cátedra es "TECNOEDU Webinars (Tecnología y Educación)" desarrolladas en el mes de diciembre de 2020. Estos seminarios en línea, retransmitidos en abierto, se presentaron con el objetivo de abordar por parte de diferentes expertos y agentes implicados, cuestiones inherentes al fenómeno COVID-19, como la transformación digital de las universidades y los nuevos modelos de enseñanza o la adaptación de los centros escolares a una nueva realidad educativa. Este ciclo de encuentros estuvo conformado por tres sesiones: "El impacto de la Covid-19 en los centros educativos en Canarias"; "Universidades Canarias y Covid-19: ¿Preparadas para la transformación digital de la enseñanza?" y "Los efectos posCovid-19 en las instituciones educativas. ¿Hacia la enseñanza híbrida?”. En estos debates se contó con diferentes profesionales, entre los cuales, fueron invitados tres docentes de centros escolares; un responsable de implementación de políticas educativas e integración curricular de la tecnología a nivel regional, una experta en la gestión de proyectos educativos con tecnología; cuatro investigadores y docentes universitarios, y dos responsables de innovación docente de distintas universidades. El público que visionó en directo estos seminarios fue de 658 personas: 263 visualizaciones en la primera sesión, 213 en la segunda, y 182 en tercera sesión.

Para la obtención de los datos de esta investigación, se distribuyó un cuestionario en línea entre los asistentes, para posteriormente conocer su experiencia pasada en el momento del confinamiento y su prospectiva en relación con los cambios que la tecnología podría propiciar en los procesos de enseñanza y aprendizaje. En total, respondieron a esta encuesta 55 personas de forma voluntaria.

El objetivo que guía este trabajo es conocer el impacto en la educación del uso de la tecnología educativa, según la percepción de distintos agentes educativos durante el confinamiento y la prospectiva que, a partir de su experiencia, prevén sobre esos usos en la educación.

\section{Materiales y método.}

El trabajo se basa en un diseño descriptivo del que extraemos, de manera sistemática, la percepción de agentes educativos participantes. Para ello hemos realizado un análisis cuantitativo y cualitativo basado en un cuestionario con preguntas cerradas y abiertas. La unidad de muestreo no probabilístico, ha sido el público participante en los webinars sobre tecnología y educación, organizados por la Cátedra Fundación Mapfre de Tecnología y Educación de la Universidad de La Laguna.

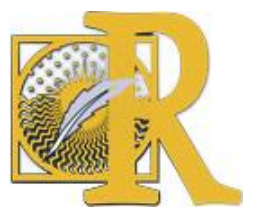


Los objetivos específicos están centrados en dos momentos, el primero, el que tuvo lugar durante el confinamiento por la pandemia de la COVID 19 y el segundo, la prospectiva que las personas participantes plantean, a medio o largo plazo, basada en la alteración de la planificación, metodología y uso de recursos. En cuanto a la descripción de los procesos educativos durante el confinamiento, nos planteamos los siguientes objetivos específicos: Conocer la disponibilidad de recursos tecnológicos; indagar sobre la disponibilidad horaria y organizativa en el trabajo de equipo; describir el esfuerzo emocional que las exigencias y tareas educativas han supuesto en este momento. En cuanto a la prospectiva, nos planteamos conocer la postura de las personas encuestadas en torno al aprovechamiento de los recursos, la flexibilidad metodológica y la propuesta de diversidad de itinerarios en una misma asignatura. Por otra parte, se pretende recoger la postura de las personas implicadas sobre la implantación de titulaciones exclusivamente online o de enseñanza presencial y semipresencial.

El instrumento aplicado es un cuestionario en línea que consta de 4 variables de identificación (sexo, tramos de edad, perfil profesional y sesión de webinar a la que ha asistido); un primer bloque con 8 preguntas dicotómicas para conocer como afectó la pandémica surgida por la COVID 19 a su experiencia educativa y una pregunta abierta para conocer los aspectos positivos y negativos de lo que supuso aprender y enseñar desde casa a través de las TIC. En el segundo bloque, que hace referencia a la prospectiva, hay 7 preguntas dicotómicas sobre cómo creen que afectarán los cambios en la práctica de enseñanza y aprendizaje, a partir de la COVID 19; se incluye una pregunta abierta para conocer las impresiones acerca de la creencia basada en el cambio y los modelos presenciales, híbridos, semipresenciales y online.

Para el análisis descriptivo de las variables dicotómicas e identificativas de la muestra, utilizamos el paquete estadístico SPSS V 25.

El análisis cualitativo de los datos está realizado mediante un sistema de codificación utilizando el programa Atlas Ti siguiendo un modelo de desarrollo de categorías inductivas (Andréu, 2002) y obteniendo un análisis de frecuencias. Una vez obtenidos los códigos y categorizados en función de los dos momentos antes descritos, hemos realizado un análisis de coocurrencia entendida como la presencia simultánea de dos códigos en un mismo espacio, estableciendo mediante este estudio, relaciones de proximidad y asociación, comprobando las estructuras de relaciones lógicas entre categorías (Escalante-Gómez, 2009). La co-ocurrencia se expresa con un valor entre 0 y 1 que indica las relaciones entre dos categorías, con respecto al total de relaciones con el resto de variables.

\section{Resultados.}

\section{Descripción de los informantes.}

Las personas encuestadas ( $n=55$ participantes) fueron un $66 \%$ mujeres, de las cuales $34,5 \%$ son menores de 34 años, el 23,7\% entre 35 y 54 años, y un 7,3\% eran mayores de 55 años. Los hombres, que conformaban el 34\% de la muestra eran en su mayoría menores de 34 años (27,3 \% del total) salvo cuatro excepciones que tenían entre 35 y 54 años.

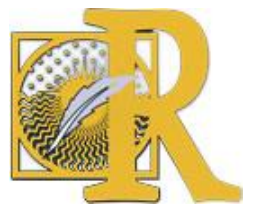




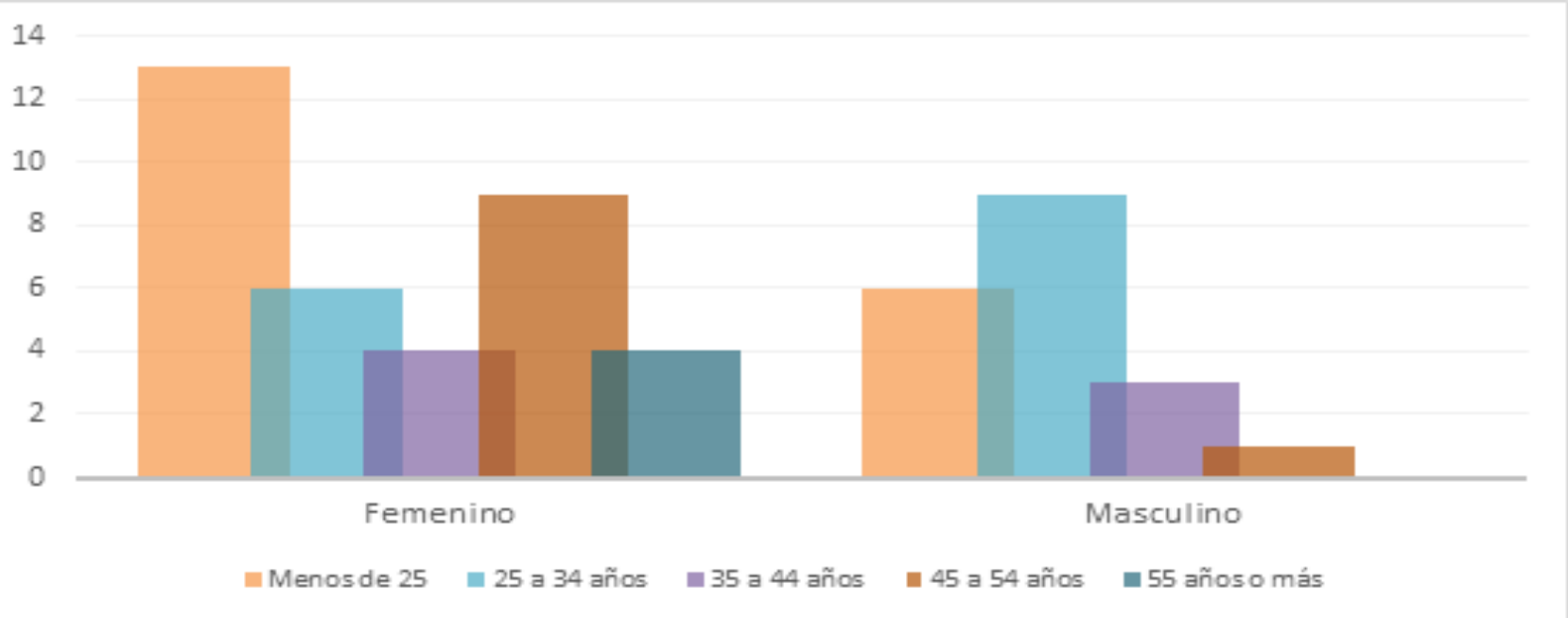

Figura 1. Descripción de los informantes.

En cuanto al perfil profesional, existe un mayor porcentaje de alumnado de doctorado, con casi un $38,2 \%$ de la muestra. Entre el $58,2 \%$ restante, encontramos que, un $21,8 \%$ eran estudiantes de grado, un 5,5\% estudiantes de máster, un 16,4\% es profesorado universitario, un $10,9 \%$ docente del ámbito educativo escolar y 3,6\% pertenecían a otras áreas profesionales de la educación.

\section{Descripción de la situación durante la pandemia surgida por la COVID.}

Comenzando con cómo afectó la pandemia surgida por la COVID a la experiencia educativa, durante el confinamiento, el $87,0 \%$ de las personas encuestadas afirman que han tenido disponibles recursos tecnológicos suficientes para poder llevar a cabo sus tareas educativas. Existe también un porcentaje elevado de personas que afirman haber podido trabajar con sus compañeros y compañeras de forma satisfactoria a través de las herramientas digitales que nos ofrece la red $(79,6 \%)$, que han obtenido mayor disponibilidad horaria y organizativa para la realización de sus tareas (72,2\%) y que, además, las personas con las que trabajan han dispuesto de recursos suficientes y buena conectividad $(74,1 \%)$.

Entre las opciones menos seleccionadas, están las que responden a efectos negativos de la pandemia en la educación. Sólo 17 personas perciben que su experiencia educativa se ha visto perjudicada por problemas de índole técnica y de conectividad, frente a los $69,1 \%$ que no han seleccionado esta opción. Esto mismo sucede con la dificultad para trasladar las tareas educativas a lo exclusivamente online. En este sentido, es necesario recordar que un $39 \%$ del total de los encuestados son estudiantes de doctorado, los cuales gozarán de mayor flexibilidad y autonomía para realizar sus tareas totalmente online. De una forma más igualada encontramos el ítem en torno al esfuerzo emocional vivenciado por el impacto de la COVID 19 en la docencia, con un 45,5\% de personas que consideran haber tenido que realizar un esfuerzo emocional mayor para responder a las exigencias y tareas educativas.

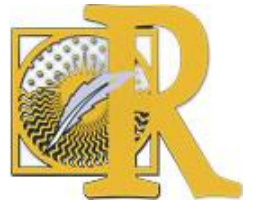


Para profundizar en la percepción vivida por los agentes educativos, hemos realizado una pregunta abierta, sin límite de palabras, basada en la experiencia como docente o como estudiante durante el confinamiento señalando los aspectos negativos y positivos de lo que supuso enseñar y aprender desde casa a través de las TIC. De esta pregunta obtuvimos, entre los resultados encontrados y el significado del contenido expresado, aspectos positivos y negativos que podemos agrupar en tres subcategorías, la primera alude a las relaciones, la segunda los aspectos educativos y curriculares y la tercera los cambios en la situación personal.

Las relaciones se han visto favorecidas por mejoras en la comunicación $(n=8)$, explicadas por la disponibilidad temporal, de recursos y conectividad para realizar consultas al profesorado, trabajos en grupo, adaptación a las situaciones personales de los demás a la hora de coordinarse y la capacidad de utilizar distintos canales y aplicaciones para la comunicación, todo ello ha influido en la satisfacción con el trabajo realizado en equipo $(n=4)$, otra de las categorías encontradas.

En esta misma subcategoría encontramos aspectos negativos que están definidos por la impersonalidad en la comunicación $(n=12)$ por medio de dispositivos móviles u ordenadores, como consecuencia de la falta de contacto directo entre profesorado-alumnado y el alumnado entre sí $(\mathrm{n}=4)$.

Las categorías o códigos que argumentan las cuestiones positivas relacionadas con elementos del currículum, las encontramos en la posibilidad de crear materiales y recursos propios $(n=2)$, por parte del profesorado y aprovechar los ya disponibles $(n=12)$, tanto por el alumnado como el profesorado. Estas dos categorías son interpretadas como una oportunidad para aprender, enseñar, relacionarse y mejorar los objetivos en el aprendizaje, que se han dado en situación de confinamiento, siendo estas valoraciones compartidas por el profesorado del ámbito escolar, universitario y por el alumnado.

Con respecto a la percepción del alumnado sobre el papel de los docentes, cabe destacar, la consideración del compromiso que han demostrado $(n=9)$. El profesorado ha señalado que se ha visto en la necesidad de mejorar su competencia digital $(n=10)$, ya que el manejo de dispositivos, uso de aplicaciones y recursos web ha sido predominante en esta etapa.

Como un aspecto positivo, dentro del currículum, se han valorado los modelos de enseñanza híbridos $(n=2)$ como un horizonte que ha quedado abierto después del confinamiento y que ha supuesto la reflexión sobre el aprovechamiento de las bondades que tiene la enseñanza y el aprendizaje presencial, virtual y a distancia.

En oposición a la connotación positiva del aprendizaje y la enseñanza en línea durante el confinamiento, encontramos valoraciones que tienen que ver con la necesidad de mejora de la competencia digital docente $(n=5)$, en el ámbito universitario y escolar y la falta de apoyo en el aprendizaje percibido del profesorado universitario $(n=5)$.

Otros profesionales de la educación y el profesorado del ámbito escolar han señalado como una categoría negativa, la dificultad en la gestión de recursos $(n=2)$, que se ha mantenido en la presencialidad de la enseñanza, después del confinamiento.

El traslado de la docencia presencia a online $(n=11)$ se ha considerado negativo porque ha resultado inesperado, por lo tanto, no se contaba con los recursos ni, en algunos casos, la formación necesaria. Entre el profesorado en general, hubo quienes fueron capaces de

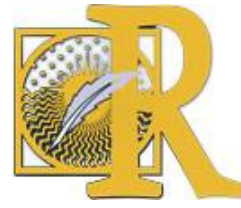


adaptarse a la nueva situación y rediseñar las clases de manera fructífera, pero también se dio el caso de profesorado que no disponía de las herramientas para afrontar el cambio. El profesorado ha señalado que el paso de la docencia presencial a la totalmente online, requiere de pequeños cambios y adaptaciones que no se pueden contemplar de la noche a la mañana.

En el plano personal hemos encontrado valoraciones que están representadas por las categorías de la mejora del aprovechamiento del tiempo $(n=8)$, la autonomía $(n=3)$ y la productividad $(n=9)$. Como aspectos negativos relacionados con la situación personal vivida durante el confinamiento por la pandemia, encontramos la inversión excesiva de tiempo $(n=7)$, la falta de motivación $(n=1)$, el desgaste emocional $(n=4)$ y la sobrecarga de trabajo $(n=3)$.

La brecha digital $(n=9)$ ha sido referenciada como una cuestión negativa, que se ha presentado, de manera extremada, durante el estado de confinamiento y que ha puesto de manifiesto, por un lado, las desigualdades en el acceso a los recursos y por otro lado las dificultades en el manejo de las TIC, desigualdades y dificultades ya conocidas, pero que se han visto acentuadas en este periodo. Las valoraciones en este sentido han sido expresadas por el alumnado y el profesorado de todos los ámbitos.

En el análisis de co-ocurrencia entre las valoraciones sobre la situación vivida durante el confinamiento, realizado después de extraer los códigos y obtener la frecuencia, encontramos relación entre la adaptabilidad y el aprovechamiento de los Materiales Didácticos Digitales (MDD) disponibles (0.14) y este último código con la mejora de la competencia digital $(0.15)$ y el compromiso docente con la mejora de la comunicación (0.13). Entre los aspectos negativos encontramos relación entre la sobrecarga de trabajo y el desgaste emocional $(0.17)$ y este último con la inversión excesiva de tiempo (0.22).

\section{Prospectiva en la educación después de la pandemia por la COVID 19.}

Continuando con el análisis, el 92,7\% de las personas encuestadas considera que, debido al impacto de la COVID cambiará los procesos de enseñanza favoreciendo el aprovechamiento de las herramientas digitales para la práctica de la enseñanza presencial. Otra cuestión bastante señalada es que se favorecerá la flexibilidad metodológica y didáctica en las próximas experiencias educativas (con un 87,3\%). Es interesante destacar que esta percepción está más presente entre el alumnado de doctorado y de máster (frente a la percepción del profesorado universitario), más abierto y optimista del posible impacto positivo de la COVID para el cambio en los procesos de enseñanza y aprendizaje.

Las personas encuestadas abogan por una percepción más flexible de las asignaturas y titulaciones como efecto tras la situación vivenciada por la COVID 19. De este modo, casi la mitad de las personas encuestadas creen que se favorecerá la propuesta de diferentes itinerarios de aprendizaje distintos para una misma asignatura $(49,1 \%)$ y un total de $65,5 \%$ considera que tras la superación de esta situación de alarma sanitaria se ofertarán más titulaciones exclusivamente online o en modalidad de enseñanza semipresencial.

Salvo una excepción, todas las personas encuestadas creen que tras la superación de la pandemia se modificarán los procesos de enseñanza online. Ninguna persona ha marcado la opción de no tener opinión formada al respecto ni la opción de que es indiferente a la

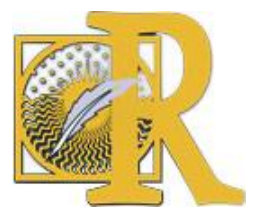


posible situación de cambio educativo en el futuro. Este dato se comprende si entendemos que nadie ha podido estar al margen de la situación producida por la COVID, aunque haya diferencias experienciales y perceptivas en base al nivel educativo y el perfil profesional.

El análisis cualitativo de la prospectiva que auguran los agentes informantes parte de la pregunta abierta: Después que pase la pandemia Covid, ¿crees que la enseñanza, sobre todo universitaria, volverá a ser totalmente presencial como antes o se producirá un cambio relevante en la misma apostando por modelos híbridos y online?

Los aspectos curriculares más relevantes que predicen los cambios postCOVID 19, son los relacionados con los modelos híbridos $(n=25)$, la mejora de la competencia digital $(n=10)$, el aprovechamiento de los recursos MDD disponibles $(n=15)$ y la adaptabilidad $(n=7)$, aspectos que ya se habían mencionado al valorar el impacto del proceso educativo durante el confinamiento. Aparecen otros elementos, relacionados con el currículum, como son: Los modelos totalmente online $(n=5)$, la mejora en la gestión de recursos $(n=3)$, la apuesta por las metodologías participativas y colaborativas $(n=4)$, la flexibilidad en la educación $(n=5)$, que hace referencia a la metodología, la innovación pedagógica y los cambios significativos. La valoración positiva $(n=2)$ que ha hecho parte del alumnado sobre la experiencia vivida, es la que le ha llevado a la conclusión de que otro modelo educativo, distinto al presencial, es posible y además creen que es una apuesta de futuro. La innovación pedagógica $(n=4)$ y los cambios significativos $(n=7)$, son otros dos códigos que definen la educación a partir de la situación de pandemia.

Para que este avance se concrete en un futuro, las personas encuestadas creen que es necesaria la mejora de la competencia digital de los docentes $(n=1)$ y un proceso amplio de reflexión sobre el traslado de la educación presencial a la que se pueda dar totalmente en línea $(n=4)$. Es preciso mencionar que el profesorado del ámbito escolar no cree que, por el momento, vayan a establecerse modelos híbridos, pero sí un mejor aprovechamiento de los MDD y los dispositivos digitales en las aulas y en los hogares.

Se mantiene la valoración negativa de la falta de contacto directo entre el profesorado y el alumnado $(n=1)$. Igualmente, en un nuevo posible escenario educativo en el que predominen modelos híbridos, el alumnado y profesorado encuestado valoran positivamente la autonomía $(n=1)$, productividad $(n=1)$ y mejora del aprovechamiento del tiempo $(n=3)$.

El análisis de co-ocurrencia entre los códigos que definen la prospectiva que hacen las personas encuestadas encontramos una relación del código descrito como modelos educativos híbridos con los cambios significativos en la educación (0.13). Los modelos educativos en línea se relacionan, según la aportación de los informantes, con la flexibilidad (0.25) y con los modelos híbridos (0.14). A partir de la situación vivida durante el confinamiento, que ha supuesto entre otros aspectos mayor utilización de los materiales digitales y de las TIC a disposición de la educación, en el análisis de contenido encontramos una relación considerable entre la presencialidad y el aprovechamiento de los recursos y MDD disponibles (0.24), fundamentalmente entre los docentes del ámbito escolar, lo que nos hace pensar, que a la vuelta a la nueva normalidad, se ha intensificado el uso de estos materiales con respecto a etapas anteriores. Los modelos presenciales también se relacionan con el código que define al contacto directo (presencial) entre el alumnado y el profesorado $(0.17)$ y las metodologías participativas y colaborativas $(0.18)$.

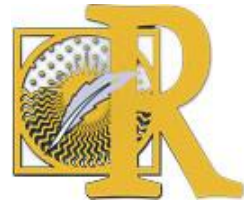


Otro hallazgo importante en el análisis de coocurrencia ha sido la relación entre el concepto de flexibilidad y los cambios significativos en la educación (0.22) y la autonomía y el aprovechamiento del tiempo (0.15). Estas relaciones son un pronóstico de lo que se espera que ocurra a partir de la experiencia educativa durante la pandemia y que no están relacionadas directamente con un modelo educativo específico, por lo tanto, podemos concluir diciendo que son aspectos que se mantendrán, independientemente del modelo que suceda.

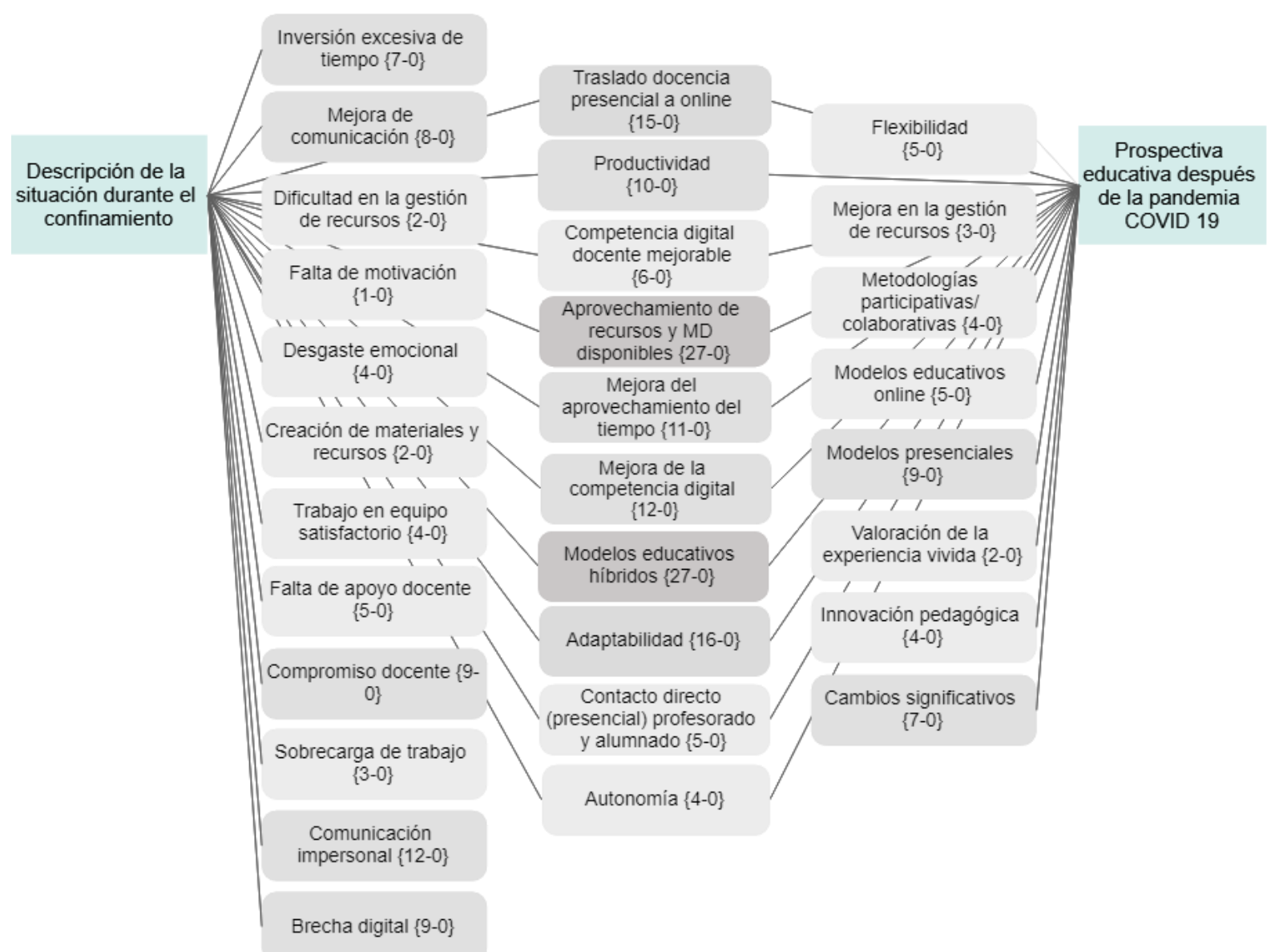

Figura 2. Códigos descriptivos de la situación durante el confinamiento y la prospectiva después de la pandemia.

\section{Discusión.}

Tras abordar los resultados de este estudio, presentamos a modo de discusión las conclusiones y principales percepciones extraídas del análisis, relacionándolas con la teoría. Entre las principales conclusiones en torno a la percepción de las personas participantes en la época del confinamiento, se destaca que la gran mayoría de las personas encuestadas afirman tener recursos disponibles para atender a tareas educativas y que han podido coordinarse con sus compañeros y compañeras de trabajo con mayor tiempo y disponibilidad

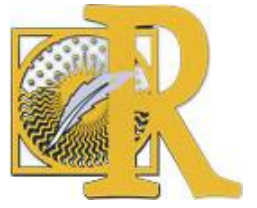

Fecha de recepción: 25-02-2021 Fecha de aceptación: 17-03-2021

Fernández-Esteban, I., Bethencourt-Aguilar, A., Martín-Gómez, S., Becerra-Brito, C. \& Area-Moreira, M. (2021). Percepciones sobre el impacto educativo de la COVID-19: Análisis de un Webinar de la 
horaria. Por el contrario, el aspecto negativo más destacable debido al impacto de la pandemia ha sido el esfuerzo emocional percibido para adecuarse a las exigencias y responsabilidades en una situación de cambio abrupto. Estas apreciaciones coinciden con lo expuesto por García-Peñalvo y Corell (2020), afirmando que el profesorado ha descubierto que el esfuerzo del docente cuando se adopta una metodología en línea es muy elevado respecto a la docencia presencial. También afirma cómo las comisiones académicas de los títulos tuvieron que llevar a cabo un importante esfuerzo de coordinación. En este sentido, es de considerar cómo la experiencia vivida durante el confinamiento ha hecho que el profesorado redescubriera, y en muchos casos fuera consciente por primera vez, de las demandas que supone la educación apoyada en la tecnología.

En cuanto al futurible tras la superación de la pandemia provocada por la COVID 19, las personas encuestadas creen que se favorecerá la flexibilidad y la oferta de titulaciones en modalidades semipresenciales u online, entendiendo que los planes de estudio de las titulaciones académicas pueden sufrir cambios tras la vivencia de una situación de incertidumbre donde la flexibilidad metodológica y didáctica ha sido clave para la reconversión de la docencia presencial a una exclusivamente online. Entendiendo esta cuestión como positiva, también existe un porcentaje alto de personas (casi la mitad) que consideran que las asignaturas también ofertarán itinerarios de aprendizaje flexibles en diferentes modalidades y propuestas didácticas. Es interesante rescatar en este punto el trabajo de Sousa-Santos, Peset-González y Muñoz-Sepúlveda, (2020), el cual, atiende al desarrollo de experiencias de aprendizaje híbrido en la educación superior, como ya se introdujo en la introducción de este trabajo. Estos resultados también se relacionan con lo extraído de la encuesta aplicada. Puede entenderse que, tras haber vivido la experiencia de una formación completamente en línea, el alumnado puede estar más predispuesto a cursar titulaciones a distancia o basadas en un modelo mixto. Expertos en investigaciones recientes de este tipo sostienen cómo este fenómeno ha de suponer un punto de inflexión en el diseño curricular de las titulaciones en educación superior, ofertando planes flexibles en cuanto tiempo y espacio (De Obesso y Nuñez, 2020).

Es de destacar que las personas encuestadas no mencionan aspectos negativos cuando se plantean la prospectiva postCOVID19, cuestiones que sí se resaltan para la fase del confinamiento. Una de estas categorías presentes en el discurso en torno al impacto de la COVID es la brecha digital. Esta variable se muestra como una barrera clave en el ejercicio de la docencia online, más presente en la opinión del profesorado perteneciente al sistema educativo escolar, posiblemente más sensible de la situación que afecta a las familias. No obstante, no se menciona este concepto ni ningún otro aspecto negativo cuando se identifica las variables pertinentes al futurible posCOVID. Este optimismo en el planteamiento futuro muestra una percepción de superación de la pandemia y de las situaciones que han asolado el proceso de enseñanza y aprendizaje que influyen en la concepción futura positiva en la docencia en la que, tras haber experimentado la ruptura abrupta del proceso de enseñanza y aprendizaje tradicional, genera una percepción en torno al futuro educativo hacia nuevas modalidades de enseñanza y mejora en la calidad del proceso educativo, entre otras. Independientemente de ello, y considerando que pueden ser muchas las razones que influyen

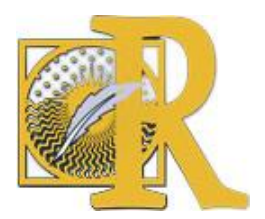

Fecha de recepción: 25-02-2021 Fecha de aceptación: 17-03-2021

Fernández-Esteban, I., Bethencourt-Aguilar, A., Martín-Gómez, S., Becerra-Brito, C. \& Area-Moreira, M. (2021). Percepciones sobre el impacto educativo de la COVID-19: Análisis de un Webinar de la 
en esa percepción, es conveniente seguir profundizando en el análisis del efecto (impacto y superación) de la COVID 19 en el sistema educativo.

A pesar de estos puntos a favor, es claramente apreciable como en el sistema educativo, en todas sus etapas, queda aún un largo camino por recorrer en lo referente a la superación de la brecha digital, ya que esto supone un importante reto necesario para alcanzar una transformación educativa sólida y sostenible en el tiempo. Aunque las personas encuestadas no aluden a la brecha digital en el futuro, siendo un foco de preocupación mientras se desarrolló la docencia online durante la pandemia, parece evidente pensar que si no se consigue superar las dificultades en la formación del profesorado y de las familias y tener accesibilidad de los recursos y la conectividad, en definitiva, superar las barreras que impiden que la transformación digital en la educación sea efectiva, difícilmente se puede pensar en el futuro en la implantación de modelos flexibles e híbridos que se han planteado en estas sesiones. En este sentido los resultados coinciden con las aportaciones de Cabero y Valencia (2020) al afirmar que la formación del profesorado es un elemento que contribuye a mitigar los efectos de la brecha digital.

Son muchos los aspectos que se han puesto de manifiesto durante el confinamiento y que de manera negativa o positiva se han valorado por parte de los agentes implicados, algunos de ellos se espera que se mantengan en el tiempo y formen parte de los beneficios de los modelos educativos en el futuro, como por ejemplo la autonomía del alumnado, la productividad y la adaptabilidad; la mejora del aprovechamiento del tiempo y de los recursos disponibles. Estas propiedades son las que se espera que formen parte de los modelos híbridos y flexibles que se perfilan en un futuro próximo. No sin tener en cuenta aquellas características no tan positivas que se han valorado como la necesidad de mejorar la competencia digital. La experiencia educativa desencadenada en los meses de marzo a junio del 2020, ha supuesto un motor de cambio y un acelerador de los procesos hacia la transformación digital, que en otras circunstancias hubiera tardado años en producirse. En un plano optimista, hay que interpretar la adaptación de la enseñanza y del aprendizaje durante el confinamiento como una oportunidad de cambio. Tomando una perspectiva positiva, se puede entender este fenómeno como un "acelerador" de un proceso de transformación digital de la docencia y de las instituciones educativas, que en muchos casos se ha venido desarrollando con lentitud. También se ha visto reflejado como esta circunstancia sobrevenida ha hecho aumentar significativamente la formación docente en el manejo de herramientas digitales para la docencia. Como bien señala García-Peñalvo y Corell (2020), se ha logrado con la pandemia en pocos meses, lo que años de cursos de formación de profesorado en competencias digitales no han logrado: una alta demanda por formarse y saber manejarse entre espacios virtuales. Para finalizar, es necesario destacar que tras analizar los datos obtenidos y extraer conclusiones, subyacen otras cuestiones a abordar en futuras investigaciones. Es de interés atender al desarrollo del curso académico 2020-2021, con la premisa de que los agentes hayan vivenciado y descubierto las posibilidades de un sistema en línea o de las tecnologías digitales como apoyo a la docencia. Es evidente las necesidades de estudio y análisis en torno a estas cuestiones.

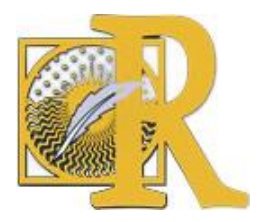




\section{Financiamiento.}

Este estudio se ha llevado a cabo como actividad de investigación y transferencia del conocimiento dentro de la Cátedra Fundación MAPFRE Guanarteneme de Tecnología y Educación de la Universidad de La Laguna (TECNOEDU).

\section{Referencias bibliográficas.}

- Andreu, J. (2002). Las técnicas de análisis de contenido. Sevilla: Fundación Centro de Estudios Andaluces. Recuperado de: https://www.centrodeestudiosandaluces.es/publicaciones/tecnicas-de-analisis-decontenido-una-revision-actualizada

- Burgos Videla, C., Vázquez-Cano, E., López-Meneses, E. y Adaos Orrego, R. (2020). DIFPRORET PROJET: Analysis of educational difficulties, proposals and challenges facing the COVID-19. IJERI: International Journal of Educational Research and Innovation, (15), 17-34. recuperado de https://doi.org/10.46661/ijeri.5145

- Cabero, J. y Barroso, J. (coords.) (2015). Nuevos retos en tecnología educativa. Madrid: Síntesis.

- Cabero, J. y Valencia, R. (2020). And COVID-19 transformed the educational system: reflections and experiences to learn. IJERI: International Journal of Educational Research and Innovation, (15), 218-228. Recuoeradi de https://doi.org/10.46661/ijeri.5246

- De Obesso, M. y Nuñez, M. (2020). El modelo educativo híbrido: una respuesta necesaria de la enseñanza universitaria a partir de la Covid-19. Recuperado de: https://www.researchgate.net/profile/Margarita-Nunez-

Canal/publication/348755808 El modelo educativo hibrido una respuesta necesaria de la ensenanza universitaria a partir de la Covid-19/links/600f52c792851c13fe39bcb6/El-modelo-educativo-hibrido-unarespuesta-necesaria-de-la-ensenanza-universitaria-a-partir-de-la-Covid-19.pdf

- Escalante-Gómez, E. (2009). Una nota metodológica sobre los análisis cualitativos. El análisis de las relaciones entre los elementos: el análisis de frecuencias y co-ocurrencias. Theorema, 18 (1), 57-67. Recuperado de:https://www.redalyc.org/articulo.oa?id=29911857006

- García-Peñalvo, f. J.; fidalgo-blanco, Á. y Sein-Echaluce, M. L. (2018). An adaptive hybrid MooC model: disrupting the MooC concept in higher education. Telematics and Informatics, 35, 1018-1030. Recuperado de: https://doi.org/10.1016/j.tele.2017.09.012

- García-Peñalvo, F.J. y Corell. A. (2020). La COVID-19: ¿enzima de la transformación digital de la docencia o reflejo de una crisis metodológica y competencial en la educación superior?. Campus Virtuales, 9(2), 83-98. Recuperado de: h74ttp://uajournals.com/ojs/index.php/campusvirtuales/article/view/740 
- Kuric Kardelis, S.; Calderón-Gómez, D. y Sanmartín Ortí, A. (2021). Educación y brecha digital en tiempos del COVID-19. Perfiles y problemáticas experimentadas por el alumnado juvenil para afrontar sus estudios durante el confinamiento. Revista de Sociología de la Educación-RASE, $14 \quad$ (1), 63-84. Recuperado de: http://dx.doi.org/10.7203/RASE.14.1.18265

- Sousa-Santos, S.; Peset-González, M.J. y Muñoz-Sepúlveda, J.A. (2020). La enseñanza híbrida mediante flipped classroom en la educación superior. Revista de Educación, 391, pp. 123-147. Recuperado de: http://www.educacionyfp.gob.es/revista-deeducacion/numeros-revista-educacion/numeros-anteriores/2021/391/391-5.html 